\title{
Diffraction Radiation by a Charge Sheet Moving past a Conducting Wedge
}

\author{
H. Henke \\ Technical University, EN-2 \\ D-1000 Berlin 10
}

\begin{abstract}
The diffraction radiation is caculated for a charge sheet moving past a conducting wedge with speed of light. 'The two-dimensional problem is analyzed in the time-domain using the conical flow method which has been developed for supersonic aerodynamics. The method transforms the wave problem into a two-dinensional potential problem which subsequently is solved by conformal mapping. Field lincs are shown for different geometries.
\end{abstract}

\section{Introduction}

The interaction between relativistic charges and environment is a problem of great practical importance and intelectual interest. For simple geometries like semi-infinite pipes, pill-box arrangements, apertures in screens and so on many analytical approaches are known. Practically all are in the frequency domain where a good insight in the physical process is difficult to be gained. On the other hand, time domain solutions are possible only in a few rare cases when complete sets of eigenfunctions exist. This is referred to as the method of Condon [1]. It has been used by several authors, e.g. [2], to calculate the wakefields of a charge passing a pill-box cavity or the gap between two intinite plates [3]. For a point charge excitation it was found that the fields are confined to delta-function like wavefronts. In a later paper [4] the results of [3] were used in order to guess the fields of a point charge passing through an aperture in an infinite plate. Only recently, since numerical codes are available, it was realized, that in case of short charges passing through apertures the fields are not confined to wavefronts but spread out over the entire volume of the velocity of light body.

The present paper treats rigorously the plane problem of a charge sheet or a line charge moving past a conducting wedge. The charge moves with velocity of light. Due to the "conical" character of the ficlds, i.e. they are independent of radial distance in $x y t$-space, a separation of the wave equation is possible and the fields can be obtained as an explicit closed expression of elementary functions. This method is called "conical flow method" and was developed by Busemann [5] for supersonic aerodynamics. Later Kel-
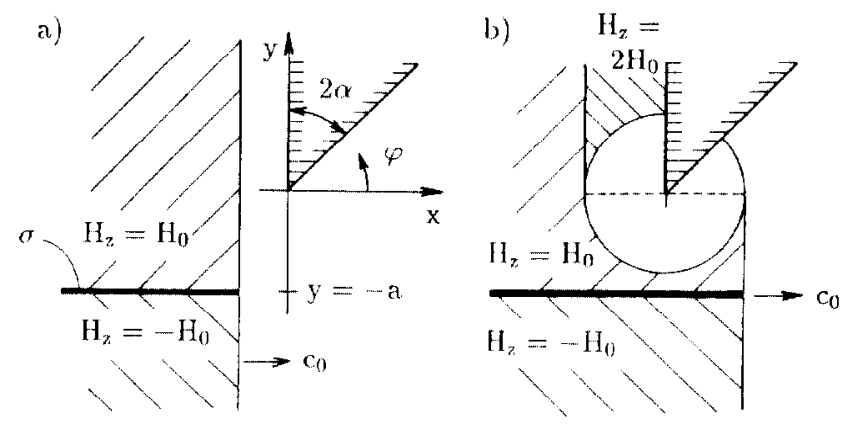

c)

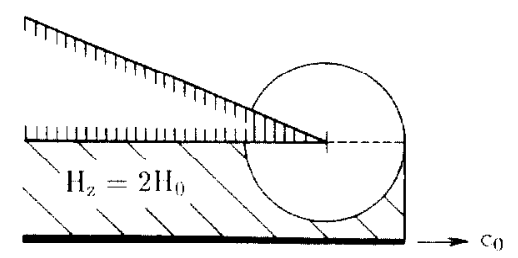

Figure 1: Sheet of charge passing a conducting wedge; a) before, b) after the wave front has reached the wedge; c) charge parallel to the wedge.

ler and Blank [6] applied the conical flow method to treat the shock diffraction of acoustic pressure or electromagnetic pulses by rigid wedges.

\section{Description of the Method}

A sheet of charge with a surface density $\sigma$ moves parallel to the $x$-axis with velocity of light. An ideal conducting wedge of opening angle $2 \alpha$ is placed in a distance $a$, Fig 1 . In order to explain the procedure we only discuss the case of Fig. 1a,b. The mathematical treatment for the case in Fig. 1c is analogous.

The magnetic field of the charge is constant above and below the charge

$$
\mathbf{H}= \pm H_{0} \mathbf{e}_{z}= \pm \frac{1}{2} \sigma c_{0} \mathbf{e}_{z}
$$

as long as the wave front has not reached the wedge yet. In front of the charge the fields are zero. When the wave front reaches the wedge a reflected and a diffracted field 
originate, Fig. 1b. The reflected adds to the incoming yielding $2 H_{0}$. The diffracted field is a cylindrical wave, where the wave front, called characteristic surface,

$$
\psi=\varrho-c_{0} t=0 \quad, \quad \varrho=\sqrt{x^{2}+y^{2}}
$$

satisfies the characteristic equation

$$
\psi_{x}^{2}+\psi_{y}^{2}-\frac{1}{c_{0}^{2}} \psi_{t}^{2}=0
$$

see e.g. [7]. The field jump across the characteristic surface $[H]=H($ inside $)-H$ (outside) follows the relation

$$
\frac{[H]}{\left[H_{0}\right]}=\sqrt{\frac{\mathrm{d} S_{0}}{\mathrm{~d} S}}=\sqrt{\frac{\varrho_{0}}{\varrho}}
$$

where $d S_{0}$ and $d S$ are surface elements at positions $\varrho_{0}$ and $\varrho$ respectively. That means, the field jump across the wave front is zero, since at the wedge corner, $\varrho=0, \mathrm{~d} S_{0}=0$ and $\left[H_{0}\right]$ finite. The boundary conditions of the diffracted field are therefore

$$
H_{z}=\left\{\begin{array}{ll}
2 H_{0} & \text { for } \pi / 2 \leq \varphi<\pi \\
H_{0} & \text { for } \pi \leq \varphi<3 \pi / 2 \\
0 & \text { for } \quad 0 \leq \varphi \leq \pi / 2-2 \alpha
\end{array} \text { and } \varrho=c_{0} t\right.
$$

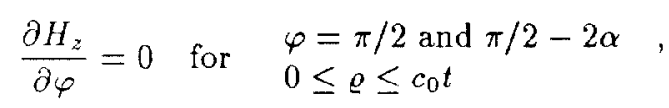

In order to solve the wave equation with boundary conditions (5) we apply the "conical flow method". The characteristic surface, equ.(2), is a cone in the $x y t$-space. Boundary values of $H_{z}$ are constant along radial lines through the origin of the cone and are also constant on the wedge. Furthermore, any radial line crosses the surface $t=\mathrm{const}$. at the same position $\varphi$ and $p=\varrho / c_{0} t$. It is therefore obvious to look for a solution $H_{z}$ within the conical sector, which is constant along each radial line. In order to do so, we introduce "normalized" cylindrical coordinates

$$
\begin{aligned}
& p=\sqrt{x^{2}+y^{2}} / c_{0} t \\
& \tan \varphi=y / x
\end{aligned}
$$

such, that the diffracted fields are within a cylindrical sector $-3 \pi / 2 \leq \varphi \leq \pi / 2-2 \alpha, 0 \leq p \leq 1,-\infty<z<+\infty$ for all instants $t$. The mapping (6) transforms the wave equation into

$$
\left(1-p^{2}\right) \frac{\partial^{2} H_{z}}{\partial p^{2}}+\left(1-2 p^{2}\right) \frac{1}{p} \frac{\partial H_{z}}{\partial p}+\frac{1}{p^{2}} \frac{\partial^{2} H_{z}}{\partial \varphi^{2}}=0
$$

which is already Laplaces equation for small $p$. With the mapping of Tschapligin [5]

$$
q=\frac{1}{p}\left(1-\sqrt{1-p^{2}}\right)=\left(\frac{1-\sqrt{1-p^{2}}}{1+\sqrt{1-p^{2}}}\right)^{1 / 2}
$$

the interior of the sector is distorted such that (7) becomes Laplacian in the whole sector where $0 \leq q \leq 1$. The wave

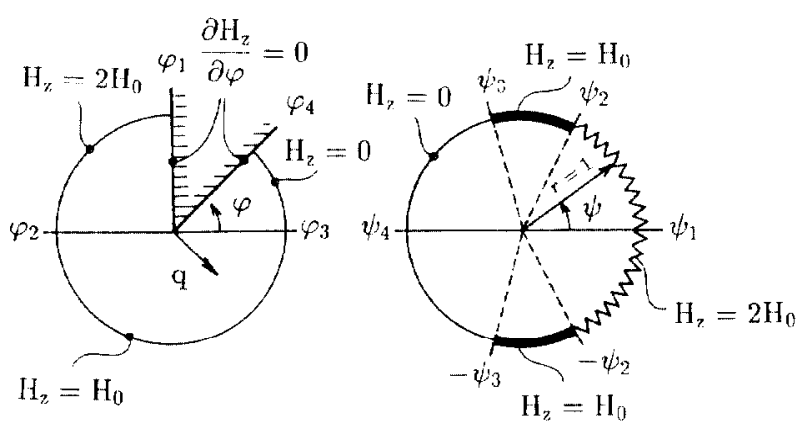

Figure 2: Mapping of the cylindrical sector in the $q \varphi$-plane onto a cylinder in the $r \psi$-plane.

problem has become a potential problem in polar coordinates $q, \varphi$ with boundary conditions (5).

A simple way to solve the problem is to map the cylindrical sector in the complex $z$-plane onto the upper half of the $w$-plane

$$
w=r \mathrm{e}^{\mathrm{j} \psi}=\left(z \mathrm{e}^{-\mathrm{j} \pi / 2}\right)^{\lambda}, \lambda=\frac{\pi}{2 \pi-2 \alpha}, z=q \mathrm{e}^{\mathrm{j} \varphi}
$$

and to complete the boundary conditions evenly on the full circle, Fig.2, such, that $\partial H_{z} / \partial \psi=0$ is satisfied on the real axis. The mapping (9) shifts planes $\varphi_{1}=\pi / 2$, $\varphi_{2}=\pi, \varphi_{3}=2 \pi, \varphi_{4}=2 \pi+(\pi / 2-2 \alpha)$ into planes $\psi_{1}=0$, $\psi_{2}=\pi \lambda / 2, \psi_{3}=3 \pi \lambda / 2, \psi_{4}=\pi$. Finally, in the $w$-plane, we have the Dirichlet potential problem for a disk with boundary conditions as shown in Fig. 2. The solution is easily found by using the Poisson integral formula [8]

$$
\begin{aligned}
H_{z}(r, \psi) & =2 H_{0} \int_{0}^{\psi_{1}}[P(r, \vartheta-\psi)+P(r, \vartheta+\psi)] \mathrm{d} \vartheta \\
& +H_{0} \int_{\psi_{1}}^{\psi_{2}}[P(r, \vartheta-\psi)+P(r, \vartheta+\psi)] \mathrm{d} \vartheta
\end{aligned}
$$

with

$$
P(r, \alpha)=\frac{1-r^{2}}{1-2 r \cos \alpha+r^{2}}
$$

After integration we find $H_{z}$ in an explicit closed expression envolving only elementary functions

$$
\begin{aligned}
& \pi \frac{H_{z}(r, \psi)}{H_{0}}= \\
& =2\left\{\arctan \frac{\left(1-r^{2}\right) \sin \frac{\pi \lambda}{2}}{\left(1+r^{2}\right) \cos \frac{\pi \lambda}{2}-2 r^{2} \cos \psi}+\delta_{1} \pi\right\} \\
& +\left\{\arctan \frac{\left(1-r^{2}\right) \sin \frac{\pi \lambda}{2}}{\left(1+r^{2}\right) \cos \frac{\pi \lambda}{2}-2 r \cos (\psi-\pi \lambda)}+\delta_{2} \pi\right\} \\
& +\left\{\arctan \frac{\left(1-r^{2}\right) \sin \frac{\pi \lambda}{2}}{\left(1+r^{2}\right) \cos \frac{\pi \lambda}{2}-2 r \cos (\psi+\pi \lambda)}+\delta_{3} \pi\right\}
\end{aligned}
$$



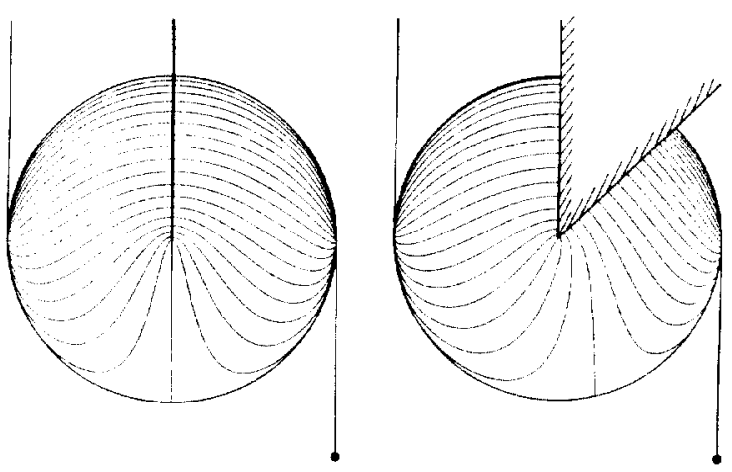

Figure 3: Lines of constant $H_{z}$ for the problem of Fig. 1a,b

where we have to take

$$
\delta_{i}=\left\{\begin{array}{lll}
0 & \text { if the argument of arctan is } \begin{array}{l}
>0 \\
1
\end{array} \quad 00
\end{array}\right.
$$

Equ. (11) together with (9), (8), (6) gives the diffracted field of the problem in Fig. 1b.

The problem of Fig. $1 \mathrm{c}$ is solved in an completely analogous way with the boundary conditions as illustrated. The result for this case is

$$
\begin{aligned}
& \pi \frac{H_{z}(r, \psi)}{H_{0}}= \\
& =2\left\{\arctan \frac{\left(1-r^{2}\right) \sin \pi \lambda}{\left(1+r^{2}\right) \cos \pi \lambda-2 r \cos \psi}+\delta \pi\right\}
\end{aligned}
$$

\section{Results}

The equations (11) and (12) determine the fields in the normalized circular sector $Q / c_{0} t \leq 1$. They are valid for times $0 \leq t \leq a / c_{0}$. For $t>a / c_{0}$ the cylindrical wave crosses the charge sheet and a constant field $-2 H_{0}$ has to be added in the segment below $y=-a$. Figures 3 and 4 show lines of constant $H_{z}$ of the diffracted wave. They are field lines of the displacement current in case of the charge sheet excitation. Since $E$ and $D$ are related by a time derivative in the same way as charge sheet and line charge are related, the field lines show also $E$-fields in case of a line charge excitation as indicated in the figures. The $H$-field is constant outside the diffraction cylinder and indicated in Fig. 1. Obviously, it is zero in front of the pulse and $\pm H_{0}$ or $2 H_{0}$ elsewhere. Points, where either the incident pulse front or the reflected pulse are tangent to the diffraction cylinder, are singular points with undefined field direction.

As can be seen in the figures, the diffracted fields and the induced charges on the surface are spread out for a $\delta$-function excitation. They are not confined to the wave front and differ therefore completely from the case where a line charge would exit from a conducting plane similar to the problem of ref. [3].

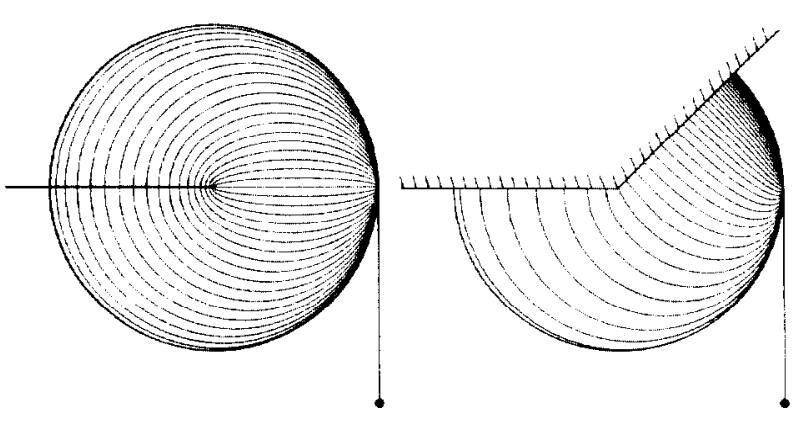

Figure 4: Lines of constant $H_{z}$ for the problem of Fig. 1c.

If the wedge degenerates to a plate, i.e. $\alpha=0$, the diffraction fields are symmetrical with respect to the two sides of the plates. That means the induced charges are symmetrical too. The total charge on one side of the plate, whichever side we consider, is equal to half the exciting charge.

\section{References}

[1] E.U. Condon, "Forced oscillations in cavity resonators", J. Appl. Phys. 12, 1941, pp. 129-132

[2] G. Dôme, "Wake potentials of a relativistic point charge crossing a bcam-pipc gap: an analytical approximation", IEEE Trans. on Nuclear Science, NS-32, $\underline{\underline{D}}$, 1985, pp. 2531-2534

[3] A.W. Chao and P.L. Morton, "Physical picture of the electromagnetic fields between two infinite conducting plates produced by a point charge moving at the speed of light", SLAC, Stanford University, report PEP-105, 1975

[4] A.W. Chao, "On the electromagnetic field produced by a point charge passing through a hole in an infinite conducting plate", SLAC, Stanford University, report PEP-Note-332, 1980

[5] A. Busemann, "Infinitesimale kegelige Überschallströmung", Schriften der Deutschen Akademie für Luftfahrtforschung, Vol. 7B, No. 3, 1943, pp. 105-121

[6] J.B. Keller and A. Blank, "Diffraction and reflection of pulses by wedges and corners", Communications on Pure and Appl. Mathematics, IV, No. 1, 1951, pp. 7594

[7] R.K. Luneburg, "Mathematical theory of optics", University of California Press, Berkeley and Los Angelos, 1964

[8] R.V. Churchill and J.W. Brown, "Complex variables and applications",McGraw-Hill, New York, 1984 